INPLASY

PROTOCOL

To cite: Li et al. The relationship between fibular fixation and tibial healing in tibiofibular shaft fractures. Inplasy protocol 2021120061. doi:

10.37766/inplasy2021.12.0061

Received: 12 December 2021

Published: 12 December 2021

Corresponding author: Li Chen

bbyxylch@126.com

Author Affiliation:

The First Affiliated Hospital of Anhui University of Science and Technology.

Support: None.

Review Stage at time of this submission: Preliminary

searches.

Conflicts of interest:

None declared.

\section{The relationship between fibular fixation and tibial healing in tibiofibular shaft fractures}

\author{
Li,C1; Dong, B2; Chen,T33 Xu, GW4.
}

Review question / Objective: Participants including healthy adults aged 18 and tibiofibular fracture caused by trauma, exclusion of intra-articular fractures, treatment with open reduction and plate fixation, closed reduction and intramedullary nail fixation, compare phil backbone and tibia fracture fixed or not dry healing the relationship, including malunion, delayed union, the relationship between postoperative complications such as infection.The surgical management of extra-articular mid and distal tibia fractures has primarily focused on reducing rates of non-union and malunion, preserving hip-knee-ankle alignment and improving functional outcomes. Fibular fractures commonly accompany these injuries and the contributory role of fixation of these fractures has been increasingly studied. A systematic review and meta-analysis were performed to determine whether concurrent fibular fixation (FF) during extra-articular mid and distal tibia fracture fixation (AO/OTA 42 and 43-A) altered the risk of malunion, non-union and post-operative complications when compared to no fibular fixation (NF).

INPLASY registration number: This protocol was registered with the International Platform of Registered Systematic Review and Meta-Analysis Protocols (INPLASY) on 12 December 2021 and was last updated on 12 December 2021 (registration number INPLASY2021120061).

\section{INTRODUCTION}

Review question / Objective: Participants including healthy adults aged 18 and tibiofibular fracture caused by trauma, exclusion of intra-articular fractures, treatment with open reduction and plate fixation, closed reduction and intramedullary nail fixation, compare phil backbone and tibia fracture fixed or not dry healing the relationship, including malunion, delayed union, the relationship between postoperative complications such as infection.The surgical management of 
extra-articular mid and distal tibia fractures has primarily focused on reducing rates of non-union and malunion, preserving hipknee-ankle alignment and improving functional outcomes. Fibular fractures commonly accompany these injuries and the contributory role of fixation of these fractures has been increasingly studied. A systematic review and meta-analysis were performed to determine whether concurrent fibular fixation (FF) during extra-articular mid and distal tibia fracture fixation (AO/OTA 42 and 43-A) altered the risk of malunion, non-union and postoperative complications when compared to no fibular fixation (NF).

Condition being studied: The surgical management of mid and distal extraarticular tibial fractures has many challenges. The injury is generally the result of a high energy mechanism and coupled with the paucity of surrounding soft tissue, is at risk of unsatisfactory radiographic and clinical outcomes.

\section{METHODS}

Participant or population: Over the age of 18, healthy patients, Patients with tibiofibular diaphyseal fracture.

Intervention: Tibiofibular shaft fractures and tibial fractures were treated with open reduction and internal fixation or closed reduction and intramedullary nail internal fixation. Fibular fixation and fibular non fixation were used for comparative study Patients with tibiofibular diaphyseal fracture.

Comparator: The tibial healing and related complications were compared.

Study designs to be included: Randomized controlled trialThe tibial healing and related complications were compared.

Eligibility criteria: Tibiofibular shaft fractures were treated with internal fixation through the shaft, and whether the fibula was fixed was divided into groups.
Information sources: A systematic search of literature in the databases of MEDLINE (via OvidSP), PubMed, Embase and The Cochrane Library from the dates of inception was performed during November 2021. Furthermore, a manual search through the reference lists of all included papers was conducted for completeness. The keywords in our search strategy included "tibia", "fibula", "fixation", "open reduction internal fixation" and relevant synonyms, acronyms and MeSH terms.

Main outcome(s): Primary outcomes collected were malunion (coronal, rotational, sagittal, or unspecified) and non-union. Subgroup analysis was undertaken for different planes of malunion. Secondary outcomes were postoperative complications and rates of further surgery. Additionally, data on diabetes, open fractures and smoking history were collected for analysis.

Quality assessment / Risk of bias analysis: Two researchers independently assessed bias in included studies using the Risk of Bias in Non-randomised Studies (ROBINS-I) tool. The quality of evidence was formally assessed for each outcome using the Grading of Recommendations, Assessment, Development and Evaluations (GRADE) framework.

Strategy of data synthesis: Statistical analysis was performed using Review Manager software 5.3 (The Cochrane Collaboration, 2014) [18]. Where appropriate, outcome measures from each study were combined via meta-analysis using a random-effects model. Relative risk (RR) with $95 \%$ confidence intervals were used for the dichotomous outcomes. Alpha was set at 0.05 , and all $p$-values generated were two-sided. A sensitivity analysis was performed by excluding non-randomised studies.

Subgroup analysis: Subgroup analysis was performed according to fracture height.

Sensitivity analysis: Changing inclusion criteria (especially controversial studies), excluding low-quality studies, using 
different statistical methods/models to analyze the same data, etc.

Country(ies) involved: China.

Keywords: The keywords in our search strategy included "tibia", "fibula", "fixation", "open reduction internal fixation" and relevant synonyms, acronyms and MeSH.

Contributions of each author:

Author 1 - Li Chen.

Author 2 - Don Bin.

Author 3 - Chen Ting.

Author 4 - Xu Gaowei. 